\title{
Evolutionary conserved role of neural cell adhesion molecule-1 in memory
}

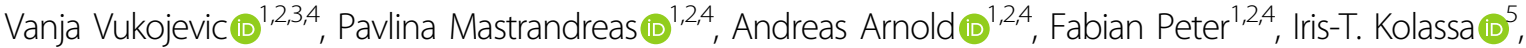 \\ Sarah Wilker, ${ }^{5,6}$, Thomas Elbert $\mathbb{D}^{7}$, Dominique J.-F. de Quervain ${ }^{1,4,8}$, Andreas Papassotiropoulos (10) ${ }^{1,2,3,4}$ and \\ Attila Stetak (iD ${ }^{1,2,3,4}$
}

\begin{abstract}
The neural cell adhesion molecule 1 (NCAM-1) has been implicated in several brain-related biological processes, including neuronal migration, axonal branching, fasciculation, and synaptogenesis, with a pivotal role in synaptic plasticity. Here, we investigated the evolutionary conserved role of NCAM-1 in learning and memory. First, we investigated sustained changes in ncam-1 expression following aversive olfactory conditioning in C. elegans using molecular genetic methods. Furthermore, we examined the link between epigenetic signatures of the NCAM1 gene and memory in two human samples of healthy individuals $(N=568$ and $N=319)$ and in two samples of traumatized individuals ( $N=350$ and $N=463$ ). We found that olfactory conditioning in $C$. elegans induced ncam-1 expression and that loss of ncam-1 function selectively impaired associative long-term memory, without causing acquisition, sensory, or short-term memory deficits. Reintroduction of the C. elegans or human NCAM1 fully rescued memory impairment, suggesting a conserved role of NCAM1 for memory. In parallel, DNA methylation of the NCAM1 promoter in two independent healthy Swiss cohorts was associated with memory performance. In two independent Sub-Saharan populations of conflict zone survivors who had faced severe trauma, DNA methylation at an alternative promoter of the NCAM1 gene was associated with traumatic memories. Our results support a role of NCAM1 in associative memory in nematodes and humans, and might, ultimately, be helpful in elucidating diagnostic markers or suggest novel therapy targets for memory-related disorders, like PTSD.
\end{abstract}

\section{Introduction}

The fundamental process of memory formation involves several steps ranging from structural and functional remodeling of synapses to changes in gene expression and de novo protein synthesis ${ }^{1-3}$. Over the last decades, it became clear that both remodeling and formation of new synapses, where neuronal cell adhesion molecules play a

Correspondence: Vanja Vukojevic (vanja.vukojevic@unibas.ch) or Pavlina Mastrandreas (pavlina.mastrandreas@unibas.ch) or

Andreas Papassotiropoulos (andreas.papas@unibas.ch)

${ }^{1}$ University of Basel, Department of Psychology, Division of Molecular Neuroscience, Birmannsgasse 8, CH-4055 Basel, Switzerland

${ }^{2}$ University of Basel, Department Biozentrum, Life Sciences Training Facility, Klingelbergstrasse 50-70, CH-4056 Basel, Switzerland

Full list of author information is available at the end of the article

These authors contributed equally: Vanja Vukojevic, Pavlina Mastrandreas

These authors contributed equally: Andreas Papassotiropoulos, Attila Stetak critical role, are essential for memory. Neuronal cell adhesion molecules of the immunoglobulin superfamily, including L1 (L1 cell adhesion molecule) and NCAM1 (neuronal cell adhesion molecule 1), are known to shape the neuronal network during development and to be involved in cognitive functions and memory in different model organisms ${ }^{4-7}$. NCAM1 protein is predominantly localized at synaptic junctions where it contributes to the modulation of neuronal activity by altering the morphology and strength of synaptic connections ${ }^{8}$. This is particularly crucial during brain development where expression of cell adhesion molecules maintains the balance between stabilization and elimination of synapses.

The NCAM1 extracellular part consists of five Ig-like and two fibronectin type III homology domains and mediates both homophilic and heterophilic interactions.

\section{(c) The Author(s) 2020}

(c) (i) Open Access This article is licensed under a Creative Commons Attribution 4.0 International License, which permits use, sharing, adaptation, distribution and reproduction c. in any medium or format, as long as you give appropriate credit to the original author(s) and the source, provide a link to the Creative Commons license, and indicate if changes were made. The images or other third party material in this article are included in the article's Creative Commons license, unless indicated otherwise in a credit line to the material. If material is not included in the article's Creative Commons license and your intended use is not permitted by statutory regulation or exceeds the permitted use, you will need to obtain permission directly from the copyright holder. To view a copy of this license, visit http://creativecommons.org/licenses/by/4.0/. 
In the vertebrate nervous system, three different forms of NCAM1 are produced by alternative splicing of a single gene (NCAM-120, NCAM-140, and NCAM-180) ${ }^{9,10}$. The three NCAM1 isoforms differ in their intracellular part and exhibit distinct expression pattern and functions ${ }^{11}$. NCAM1 has been shown to be involved in both short- and long-term synaptic plasticity ${ }^{4,12,13}$. The role of NCAM1 in memory was first proposed by a pioneering study, which demonstrated that administration of antibodies or synthetic peptides against NCAM1 inhibited the induction of $\mathrm{LTP}^{14}$. Later studies demonstrated, that NCAM1 knockout (KO) mice show severe spatial memory deficits ${ }^{15,16}$ and loss of NCAM1 impaired fear conditioning ${ }^{16,17}$. Moreover, increasing amount of evidence points to the pivotal role of NCAM1 in the acquisition and formation of emotional memories (reviewed in refs. $\left.{ }^{1-3,6}\right)$. Neuronal activity in the amygdala, a brain region centrally involved in emotional processing, is upregulated in response to stress in NCAM1 KO mice versus wild-type controls ${ }^{4-7,18}$. Moreover, NCAM1 seems to be required for fear conditioning and consolidation, as auditory and contextual fear memories were significantly impaired in NCAM1 KO mice ${ }^{8,16}$. In rats, expression of hippocampal NCAM1 changed upon emotional experience in a time- and intensity-dependent manner. Finally, exposure of rats to a traumatic event significantly impaired spatial memory formation and induced a reduction of the NCAM1 $180 \mathrm{kDa}$ isoform in the hippocampus ${ }^{9,10,19}$.

The broad range of biological effects of NCAM1 requires precise regulation of its protein activity. While post-translational modifications of NCAM1 have been widely studied, much less is known about the transcriptional regulation of NCAM1 in biological processes, including learning and memory. A recent study investigating gene expression activation during long-term associative memory (LTAM) in C. elegans identified the NCAM1 homolog as one of the genes significantly upregulated in $\mathrm{LTAM}^{20}$. In addition, the promoter region of the human NCAM1 was previously shown to contain a high proportion of CpG sites and to lack active TATA or CCAAT as transcriptional regulatory elements ${ }^{21}$, suggesting that methylation could be an important mechanism for the regulation of this gene's expression. Generally, DNA methylation seems to be centrally involved in memory coding ${ }^{22-27}$, formation as well as maintenance ${ }^{15,16,23-27}$.

In the current study, we investigated the role of neural cell adhesion molecule 1 in learning and memory in nematodes and humans. We found that ncam-1 is upregulated at the transcriptional level during a LTAM task in C. elegans. ncam-1 loss of function (lf) specifically impaired LTAM, which was fully rescued by introduction of the human NCAM1 in mutant worms, suggesting an evolutionary conserved function of NCAM1 in long-term memory. Finally, we showed an association of NCAM1 DNA methylation patterns with memory performance and traumatic memory, in healthy young individuals and conflict zone survivors, respectively.

\section{Methods and materials}

General methods and strains used

Standard methods were used for maintaining and manipulating C. elegans ${ }^{28}$ (see Supplemental Information).

\section{Targeted modification of ncam-1 using CRISPR/Cas9}

Loss of function mutant ncam-1(utr3) was generated using the CRISPR/Cas9 strategy, targeting two cleavage sites flanking the second intron of the gene (common to all three C. elegans ncam-1 isoforms, see Supplemental Information).

\section{Extrachromosomal transgenic lines}

For the C. elegans rescue experiment, ncam-1(utr3) mutant worms were injected with the $17.44 \mathrm{~kb}$ Eco47III/ KpnI digested fragment from the pCC1FOS wrm0619dG03 fosmid. For the human rescue construct, human ncam1 cDNA (encoding amino acids 1-858) was introduced under the control of a $2 \mathrm{~kb}$ C. elegans ncam-1 promoter (see Supplemental Information).

\section{C. elegans behavioral assays}

Chemotaxis to olfactory cues was tested as previously described $^{29}$. Negative olfactory conditioning was performed with diacetyl (DA) as previously described ${ }^{30}$. LTAM was tested using two cycles of conditioning with 30 min feeding without DA inbetween trainings. After the spaced training, worms were kept on NGM plates in the presence of abundant food for $24 \mathrm{~h}$ and tested for their chemotaxis toward DA after the recovery phase ${ }^{31}$. For details see Supplemental Information.

\section{Real-time quantitative polymerase chain reaction (PCR)}

Total RNA was isolated from synchronized adult worms using the Direct-zol RNA MiniPrep kit (Zymo Research Cooperation, Irvine, CA). Real-time PCR was performed with gene specific primers using the SyBr Fast kit (Kapa Biosystems, Wilmington, MA) according to the manufacturer's recommendations in a Rotor Gene-6000 instrument (Corbett Research, Mortlake, NSW). Expression levels were normalized to $t b a-1$ expression level. Fold differences were calculated using the $\Delta \Delta \mathrm{Ct}$ method $^{32}$ (see Supplemental Information).

\section{Fluorescence microscopy}

Whole worms were mounted on 3\% agar pads and immobilized with CTX buffer supplemented with sodium azide. NCAM-1::YFP animals were imaged using a Zeiss 
Axiovert 200 M LSM 5 Pascal confocal microscope equipped with a $\times 40$ oil immersion objective (see Supplemental Information).

\section{Human studies}

\section{Swiss samples, healthy young adults}

Memory was assessed in two independent samples of healthy young adults from Basel, Switzerland (Swiss Sample 1: $N=568$; mean age 23.8 y, $18.3-36.8$ y; $59 \%$ females; Swiss sample 2: $N=319$; mean age $24.1 \mathrm{y}$, $18.3-36.5$ y; $70 \%$ females). Subjects performed several different consecutive tasks as described in detail previously $^{33,34}$. For the purpose of the present study we focused on episodic memory and therefore we analyzed the emotional and neutral picture-encoding task. In the Swiss sample 1, the delayed recognition task was tested $1 \mathrm{~h}$ after encoding. In Swiss sample 2, the delayed free recall task was tested $24 \mathrm{~h}$ after encoding. For DNA isolation, blood samples were collected at the time-point of the main investigation. For details see Supplemental Information.

\section{African samples, conflict survivors}

PTSD risk and symptomatology were assessed in two independent African samples. In the African sample 1, we have investigated $N=350$ survivors from the 1994 Rwandan genocide (mean age $34.8 \mathrm{y}, 18-68 \mathrm{y} ; 49.1 \%$ females; $67.8 \%$ with PTSD lifetime diagnosis; Supplemental Table S1). For the African sample 2, we have included $N=463$ survivors of the rebel war in Northern Uganda (mean age $29 \mathrm{y}, 18-55 \mathrm{y} ; 44.1 \%$ females; 68.2\% with PTSD lifetime diagnosis; Supplemental Table S1).

All subjects had experienced traumatic situations and were examined according to DSM-IV criteria ${ }^{35}$ in the period 2006-2009 (African sample 1) and 2009 to 2011 (African sample 2). Traumatic load was estimated by assessing the number of different traumatic event types experienced or witnessed ${ }^{36}$. Taking into account known ceiling effects of trauma load on PTSD risk, individuals with extreme levels of trauma exposure were excluded for the current analyses ${ }^{37}$. Saliva samples for the DNA isolation were collected at the time-point of the main investigation (see Supplemental Information).

\section{DNA isolation from human samples}

Saliva samples were collected using an Oragene DNA Kit (DNA Genotek, Ottawa, ONT) and DNA was extracted using the precipitation protocol recommended by the manufacturer and then re-purified.

Blood samples were collected using the $10.0 \mathrm{~mL}$ BD Vacutainer $^{\circledR}$ Plus plastic whole blood tube, BD Hemogard ${ }^{\mathrm{Tm}}$ closure with spray-coated K2EDTA (Becton Dickinson, Franklin Lakes, NJ). DNA was isolated with QIAmp Blood
Maxi Kit (Qiagen AG, Hilden, Germany), using the recommended spin protocol (see Supplemental Information).

\section{Illumina human Methylation BeadChip methylation analyses}

DNA isolated from peripheral blood or saliva was investigated with the Illumina human Methylation BeadChip $450 \mathrm{~K}$ array (Swiss Samples 1 \& 2 and African Sample 1) or EPIC array (African Sample 2, restricted to the probe-set common with the $450 \mathrm{~K}$ array) (Illumina, Inc., San Diego, CA).

For preprocessing, data were extracted and analyzed using the $\mathrm{R}$ package RnBeads version $0.99 .9^{38}$. The background was subtracted using the "noob" method in the methylumi package ${ }^{39}$, and the signal was further normalized using the SWAN algorithm ${ }^{40}$. Post-processing was further done for each of the four samples separately, combining the $B$ values of the pre-processed data of all batches per sample (see Supplemental Information).

Finally, we used the genome-wide functional segmentation as specified by the ENCODE Combined chromatin states $^{41,42}$, and then calculated mean methylation values for each of 13 functional elements of the NCAM1 locus (GRCh37/hg19; rtracklayer R package ${ }^{43}$ ).

\section{Genotyping}

Single-nucleotide polymorphisms (SNP) genotyping for all samples was done with Affymetrix SNP 6.0 array platform for all four investigated samples, as previously described ${ }^{44}$. Quantitative trait loci (QTL) analysis was performed with MOLGENIS meQTL (methylation QTL) pipeline. For details see Supplemental Information.

\section{Statistical analyses}

Delayed recognition or delayed recall as dependent variable was modeled against the DNA methylation of predefined functional ENCODE elements by linear regression, taking into account the interaction with the valence of pictures used in the emotional picture-encoding task.

In the African sample, the association between lifetime post-traumatic stress disorder symptom scores as dependent variables and DNA methylation of predefined functional ENCODE elements was assessed by linear regression. To account for trauma load as a principal factor in the development of PTSD ${ }^{37}$ sum of lifetime traumatic event types was used as a covariate in the linear regression model. The relationship between DNA methylation at NCAM1 putative promoter and lifetime PTSD was assessed using binary logistic regression, with NCAM1 promoter methylation as a quantitative predictor and sum of life traumatic event types as a covariate.

Bonferroni correction was implemented to account for multiple testing procedures. The significance threshold 
was set to $P=0.05$. Statistical analyses were done in $\mathrm{R}(\mathrm{R}$ version 3.6.0; $R$ Development Core Team 2017), using the cpg.assoc $^{45}$ and $n l m e^{46} \mathrm{R}$ packages.

All human laboratory procedures were conducted in a blind, randomized order. For details see Supplemental Information.

\section{Results}

ncam-1 is transcriptionally upregulated during LTAM

In previous array-based experiments in C. elegans, ncam-1 was upregulated during LTAM formation in a temporal manner ${ }^{20,47}$. To confirm this observation, we carried out qRT-PCR and assessed gene expression in trained worms ( $4 \mathrm{~h}$ after conditioning) compared to untrained worms or animals exposed to starvation or to DA alone. We found that ncam-1 mRNA levels were significantly increased in trained animals (Fig. 1a). Furthermore, by using the temporal transcriptomic data from our recent publication ${ }^{20}$ we showed that $n c a m-1$ has a transcriptional peak $4 \mathrm{~h}$ after aversive olfactory conditioning training and remains upregulated up to $24 \mathrm{~h}$ post-conditioning (Fig. 1b).

\section{Loss of ncam-1 impairs negative olfactory long-term memory}

In order to study the physiological role of the sole C. elegans NCAM1 ortholog, we first generated a deletion in the ncam-1 gene using the CRISPR/Cas9 system (Supplemental Fig. S1). Worms were injected with a mixture containing two different sgRNAs targeting distinct cleavage sites in the $n c a m-1$ gene locus and screened for deletion in the ncam-1 gene. After screening we identified an allele carrying a deletion and simultaneous insertion that causes a frame shift in all three isoforms of the C. elegans ncam-1 (Supplemental Fig. S1). Deletion of the ncam-1 gene in worms does not result in a visible phenotype, animals appear healthy, are fertile and have no obvious morphological or locomotory defects.

In order to test the role of ncam-1 in the regulation of aversive olfactory associative learning, we first tested the chemotactic behavior of ncam-1(lf) mutant worms towards three different chemoattractants (diacetyl, isomyl-alcohol, and benzaldehyde) and a repellent (octanol) as previously described ${ }^{29}$ (Fig. 2a). Mutant worms exhibited similar chemotaxis to wild-type animals, indicating that $n c a m-1(l f)$ has no sensory defects. Next, we investigated the potential role of NCAM-1 in olfactory associative learning. In negative olfactory conditioning, untrained wild-type, and ncam-1(lf) animals exhibited strong attraction to DA to the same extent. Furthermore, after aversive olfactory conditioning with starvation in the presence of DA, both strains displayed strong repulsion toward DA, suggesting that ncam-1 is not required for memory acquisition in this model. We also tested the

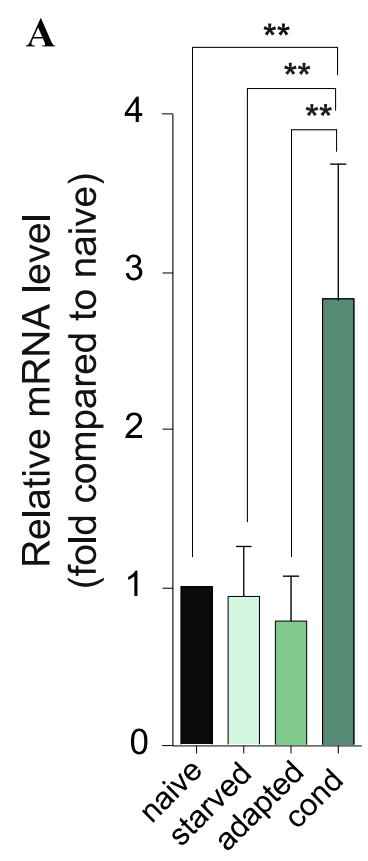

B

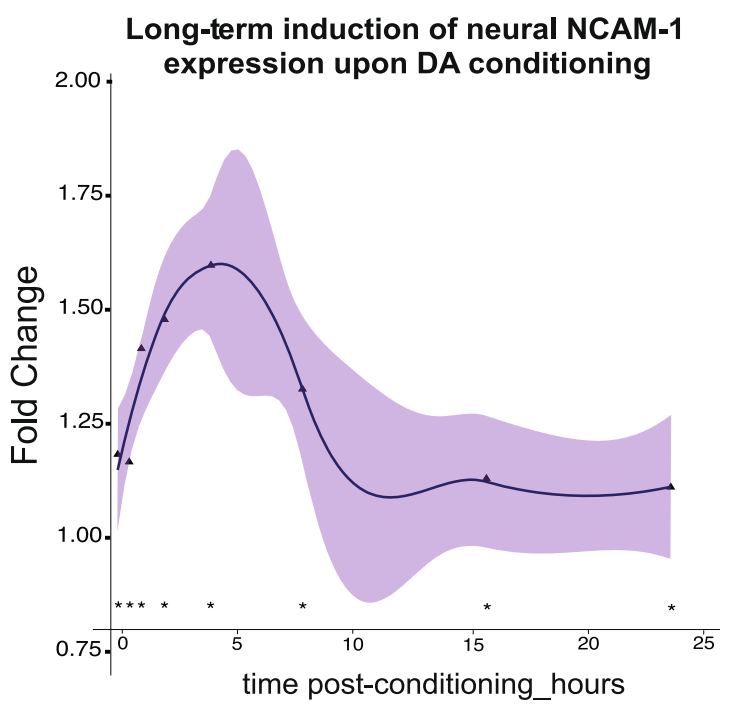

Fig. 1 NCAM-1 is upregulated during long-term memory formation. a Relative ncam-1 mRNA levels were measured using qRT-PCR from total RNA isolated from wild-type animals prior to treatment (naive), starved, DA only exposed and $4 \mathrm{~h}$ post conditioning (five biological samples each performed in triplicates). b Temporal transcriptomic analysis of ncam-1 expression up to $24 \mathrm{~h}$ upon aversive conditioning, with peak expression occurring at $4 \mathrm{~h}$ post-conditioning (three independent biological replicates ${ }^{20}$ ). 


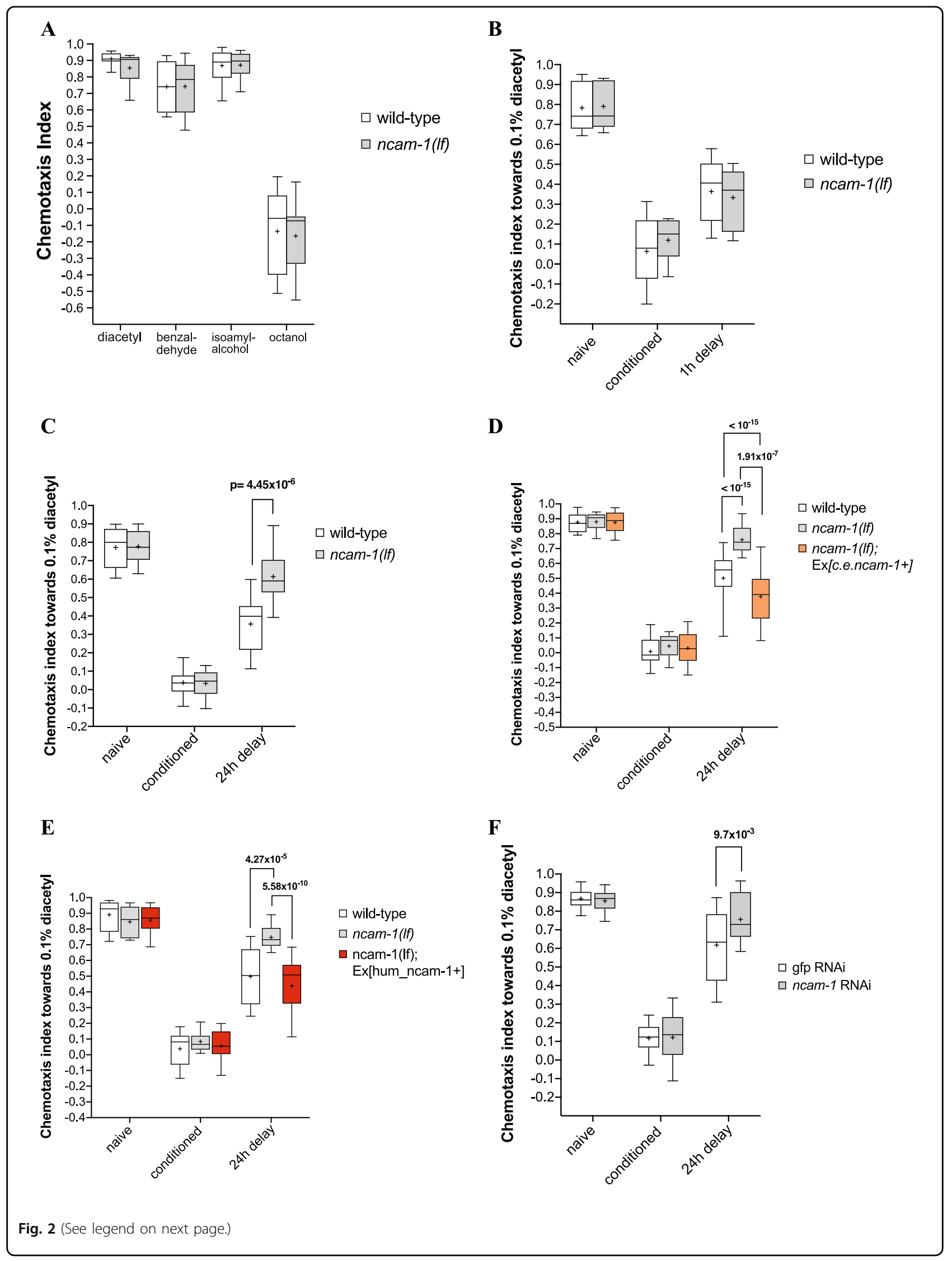


(see figure on previous page)

Fig. 2 NCAM-1 regulates aversive long-term memory. a Chemotaxis of wild-type or mutant worms was assayed towards $0.1 \%$ diacetyl, benzaldehyde, or isoamyl-alcohol volatile chemoattractant, and $0.1 \%$ octanol as repellent. Chemotaxis index was calculated as $\mathrm{Cl}=($ worms at the attractant spot-worms at the solvent spot)/total number of worms. b, c Conditioned wild-type and ncam-1(If) mutant worms were tested for their preference towards diacetyl immediately after conditioning (conditioned), after a $1 \mathrm{~h}$ ( $1 \mathrm{~h}$ delay), or after a $24 \mathrm{~h}$ delay ( $24 \mathrm{~h}$ delay). d, e LTAM DA conditioning of wild-type ncam-1 (If) worms carrying C. elegans genomic ncam-1 locus (d) or human NCAM1 CDNA (e) to DA, immediately (conditioned) or following 24-h recovery in the absence of DA (24 $\mathrm{h}$ delay). $\mathbf{f}$ LTAM conditioning of RNAi-hypersensitive worms treated with ncam-1 or control gfp RNAi from early L3 until adulthood. Worms were assayed toward DA without (naive), immediately (conditioned) or $24 \mathrm{~h}$ following DA conditioning ( $24 \mathrm{~h}$ delay). All experiments were done in triplicate and repeated at least three times. Box plots showing the 10th and 90th percentiles are presented for each condition and genotype. Statistical significance was assessed with two-way ANOVA and post hoc $t$ tests between groups as indicated (Bonferroni's adjusted $p$ values are reported).

ability of animals to retain this learnt behavior over time (short-term associative memory [STAM] or long-term associative memory [LTAM]). In STAM, nematodes were subjected to conditioning and allowed to recover for $1 \mathrm{~h}$, prior to testing for their DA preference ${ }^{48,49}$. In both wildtype and ncam-1(lf) animals, the negative association of DA with starvation persisted to a similar extent during the recovery period tested (Fig. 2b). This result indicates that NCAM-1 is not required for aversive olfactory learning and for short-term memory in C. elegans. To test for LTAM, worms were conditioned using two rounds of training with food withdrawal in the presence of DA as described and were then tested for their DA attraction after a $24 \mathrm{~h}$ delay $^{24}$. Whilst learning was similar between genotypes, a significant decrease in LTAM was observed in ncam-1(lf) mutants compared to wild-type worms (Fig. 2c). Reintroduction of a wild-type $18 \mathrm{~kb}$ genomic fragment of the ncam-1 gene (Fig. 2d) or human NCAM1 cDNA under the control of the C. elegans $5^{\prime}$ and $3^{\prime}$ regulatory elements into mutant worms fully rescued the memory defect (Fig. 2e). Finally, the effect of NCAM-1 was not due to a developmental defect, since RNAi silencing of ncam-1 following neuronal differentiation phenocopied the ncam-1(lf) phenotype (Fig. 2f).

\section{Expression of NCAM-1 in C. elegans}

To investigate the expression pattern of NCAM-1, we tagged the endogenous protein at the C-terminal end with yellow fluorescent protein (YPET) using CRISPR/ Cas9 strategy. The targeted genomic position was shared between all three ncam-1 isoforms, ensuring that all protein isoforms are tagged. To confirm the functional integrity of the tagged protein, we compared the memory performance between wild-type and NCAM-1::YPET animals and found no difference (Fig. 3a), suggesting that YPET does not alter the function of the protein. Next, we analyzed the expression of NCAM- 1 and found that the protein is expressed throughout the life cycle of C. elegans in several tissues, including the pharynx (Fig. 3b), nerve ring (Fig. 3c), in the ventral nerve cord (VNC) (Fig. 3d), germline (Fig. 3e), spermatheca (Fig. 3f), and some tail neurons (Fig. $3 \mathrm{~g})^{50}$. Since NCAM1 plays a pivotal role in synaptic formation, maturation and maintenance, we investigated synapse morphology in ncam-1(lf) worms. Specifically, we looked for changes in GLR-1 containing synapses since glutamatergic signaling has been previously shown to be essential in modulating associative memory formation in C. elegans ${ }^{51}$. By visualizing the distribution of GFP-tagged GLR-1 receptors (GLR-1:: GFP) in wild-type and ncam-1(lf) animals, we showed that in the VNC, GLR-1::GFP puncta shape and distribution appeared to be unchanged (Fig. 4a, b) and the cell bodies of head neurons showed similar GLR-1::GFP distribution (Fig. 4c, d). We further carried out quantification analyses by measuring the fluorescence intensity (Fig. 4e) and the number of puncta (Fig. 4f) along the VNC, from the vulva region all the way to the tail neuron and showed no differences between wild-type and ncam-1(lf) animals. Together, our results suggest that loss of NCAM-1 does not interfere with the distribution or number of GLR-1 receptors suggesting that our memory phenotype is indeed attributed to the loss of the protein rather than to a general synaptic defect.

\section{DNA methylation of the human NCAM1 promoter is associated with delayed recognition performance}

Next, we investigated the association between DNA methylation of the functional elements of the human NCAM1 gene locus and memory performance. We first used the Swiss sample 1, which was tested for delayed recognition of the previously seen emotional and neutral pictures. After accounting for multiple testing, we found that DNA methylation of the ENCODE-predicted NCAM1 promoter (Fig. 5) was significantly associated with the delayed recognition performance $(\beta=-0.46$, $P_{\text {uncorrected }}=0.0004, P_{\text {corrected }}<0.05$, Supplemental Fig. S2A). Furthermore, there was a significant interaction between picture valence and DNA methylation of ENCODE-predicted NCAM1 promoter $\left(F_{(2,1114)}=5.7\right.$, $\left.P_{\text {interaction }}=0.004\right)$. Post hoc analysis revealed that the DNA methylation of the NCAM1 promoter was associated with the recognition performance of negative and neutral 

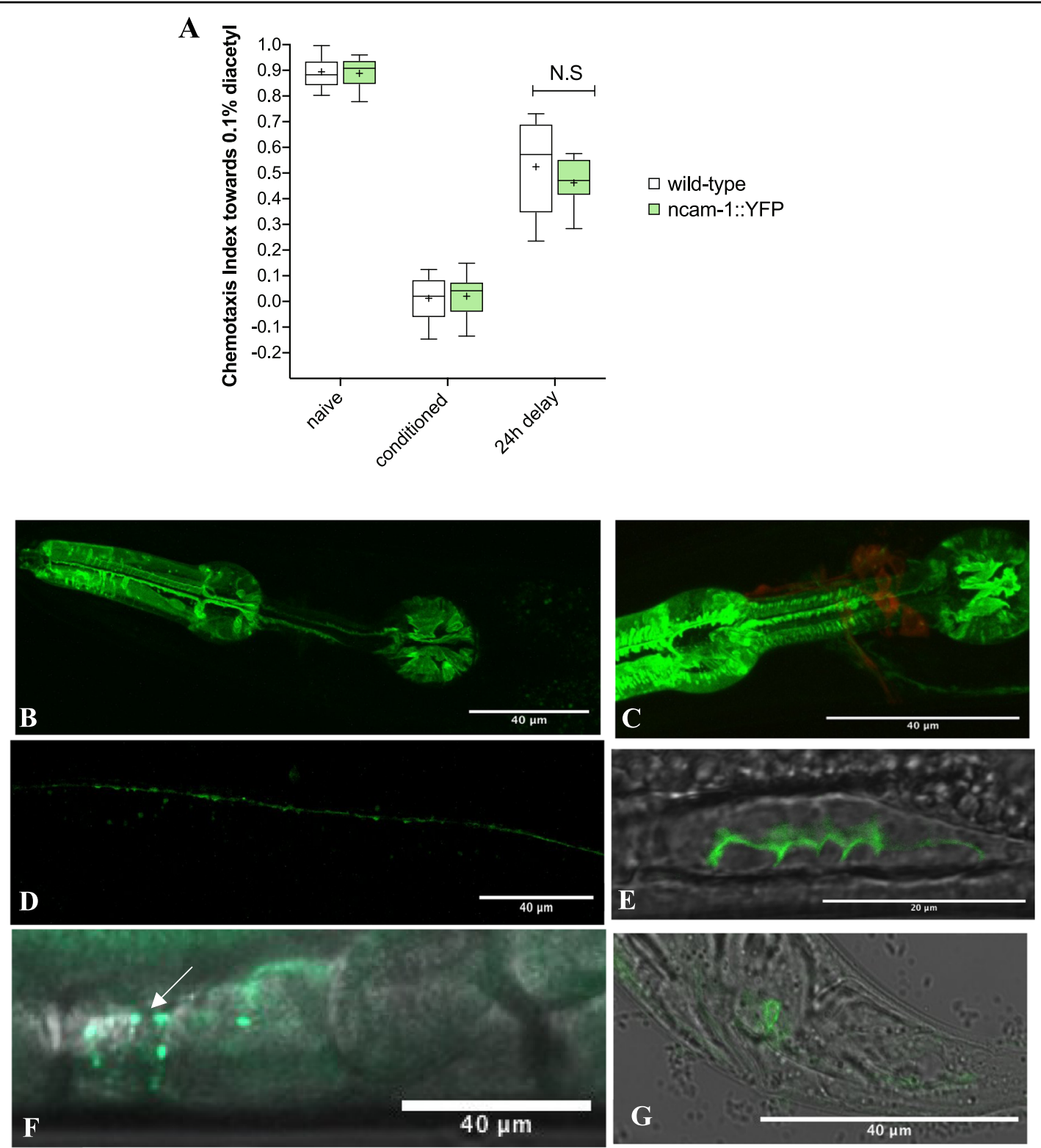

Fig. 3 Expression pattern of NCAM-1. a Conditioned wild-type and ncam-1::YPET mutant worms were tested for their preference towards diacetyl immediately after conditioning (conditioned) and after a $24-\mathrm{h}$ delay ( $24 \mathrm{~h}$ delay). All experiments were done in triplicate and repeated at least three times. Data sets were compared using two-way ANOVA. Box plots showing the 10th and 90th percentiles are presented for each condition and genotype. $\mathbf{b}$ Localization of ncam-1.:YPET expression in the pharynx, $\mathbf{c}$ in the nerve ring (ncam-1 in green and DID stained head neurons in red), $\mathbf{d}$ in the VNC $\mathbf{e}$ in the germline, $\mathbf{f}$ in the spermatheca and $\mathbf{g}$ in the tail.

content, but not with the positive content (Table 1). Finally, DNA methylation of the NCAM1 promoter was not associated with the immediate recall performance in the same emotional memory task $(P>0.05)$.

\section{DNA methylation of the NCAM1 promoter is associated with $24 \mathrm{~h}$ delayed free recall}

In order to further investigate the association between NCAM1 and remote memory performance we analyzed data of the Swiss Sample 2, in which we tested a 24-h delayed free recall of previously seen emotional and neutral pictures. Here, the DNA methylation of NCAM1 promoter was negatively associated with the delayed recall of previously seen pictures $\left(\beta=-3.0, P_{\text {uncorrected }}=0.003\right)$. The interaction between valence and DNA methylation of the ENCODE-predicted NCAM1 promoter on delayed free recall was nonsignificant $\left(F_{(2,634)}=2.8, P=0.06\right)$. However, a post hoc analysis revealed that the DNA 

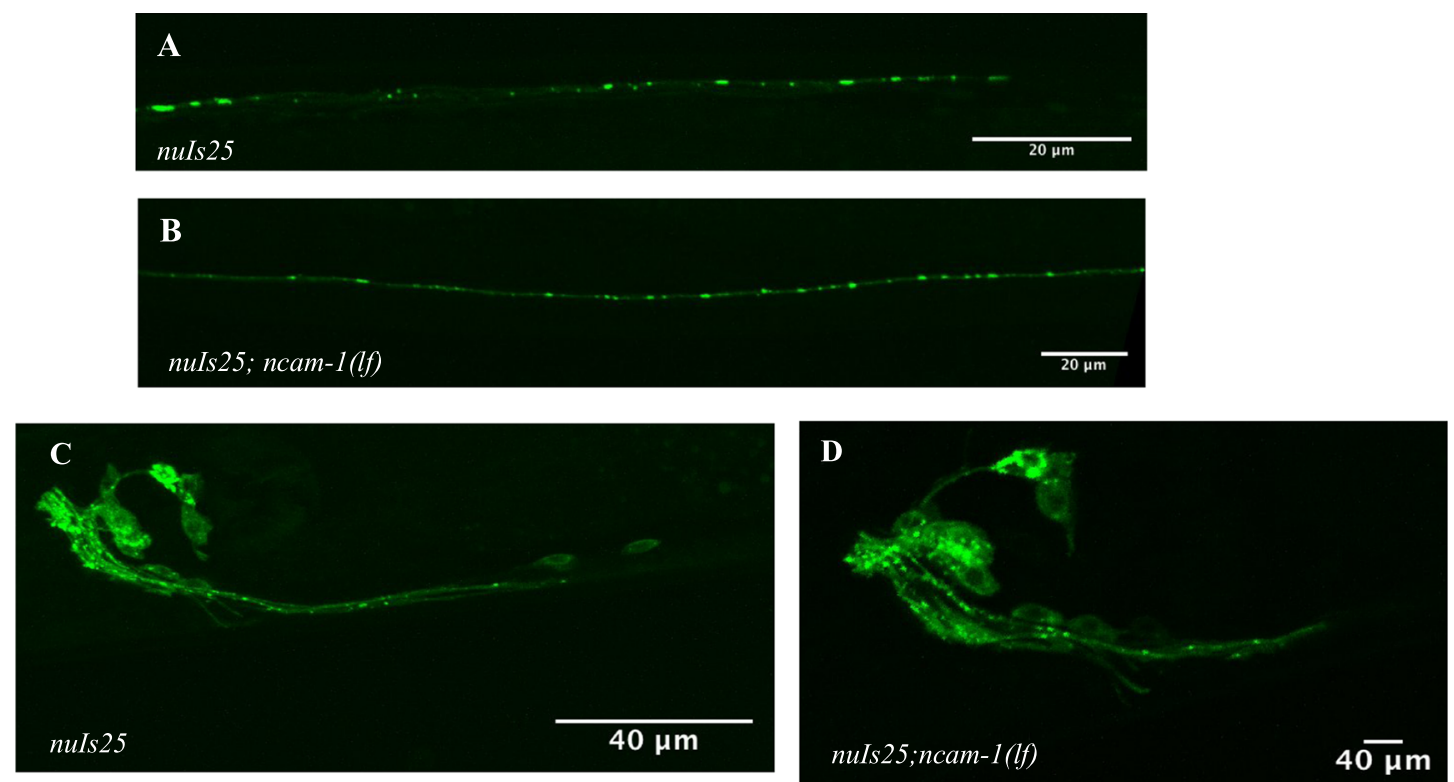

$\mathbf{E}$
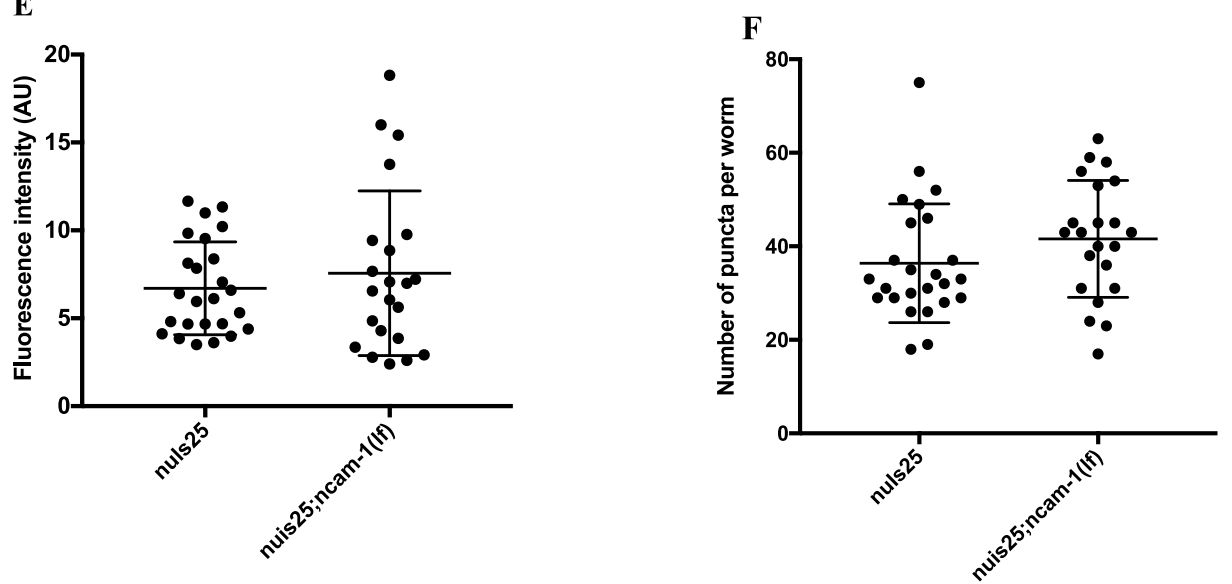

Fig. 4 Synapse morphology in GLR-1 receptor subunits remains intact in ncam-1 (If) worms. a, b Representative expression of GLR-1::GFP protein in the VNC in (a) nuls25[g/r-1::GFP] and (b) nuls25[g/r-1::GFP];ncam-1 (If) transgenic worms. Images were always captured from the vulva all the way to the tail neuron. c, d Representative expression of GLR-1::GFP protein in the head ganglia in (c) nuls25 and (d) nuls25, ncam-1(If) transgenic worms. e, $\mathbf{f}$ Scatter plots displaying fluorescent intensity values $\mathbf{e}$ and number of punctae $\mathbf{f}$ per worm with the line in the middle representing the mean and the whiskers representing \pm SD. A two-tailed unpaired Mann-Whitney $U$ test revealed no significant differences between nuls 25 and nuls25; ncam-1(If) lines.

methylation of the NCAM1 promoter was associated with the delayed free recall of negative content, but not with the positive or neutral content (Table 1).

\section{DNA methylation of the NCAM1 promoter is associated with lifetime intrusive memories}

Long-lasting aversive memories of past traumatic events are an important cognitive component of posttraumatic stress disorder (PTSD) etiology and symptomatology. Guided by the findings in the healthy samples, we subsequently analyzed data of conflict zone survivors in the two African samples. First, a comparison of methylation levels across NCAM1
Encode elements between the Swiss and African samples did not reveal significant differences in median, distribution, or variability $\left(P_{s}>0.05\right.$, see Methods). Next, given the known differences in the genetic architecture between populations of European and African descent, as well as the existence of alternative promoters of the NCAM1 gene, we first performed an explorative analysis across all functional segments of the NCAM1 locus (Fig. 5, Supplemental Fig. S2B). After accounting for multiple testing, we observed that the DNA methylation of the alternative, putative, NCAM1 promoter was negatively associated with life-time PDS symptoms (main effect - African Sample 1: $t_{(347)}=-3.0$, 


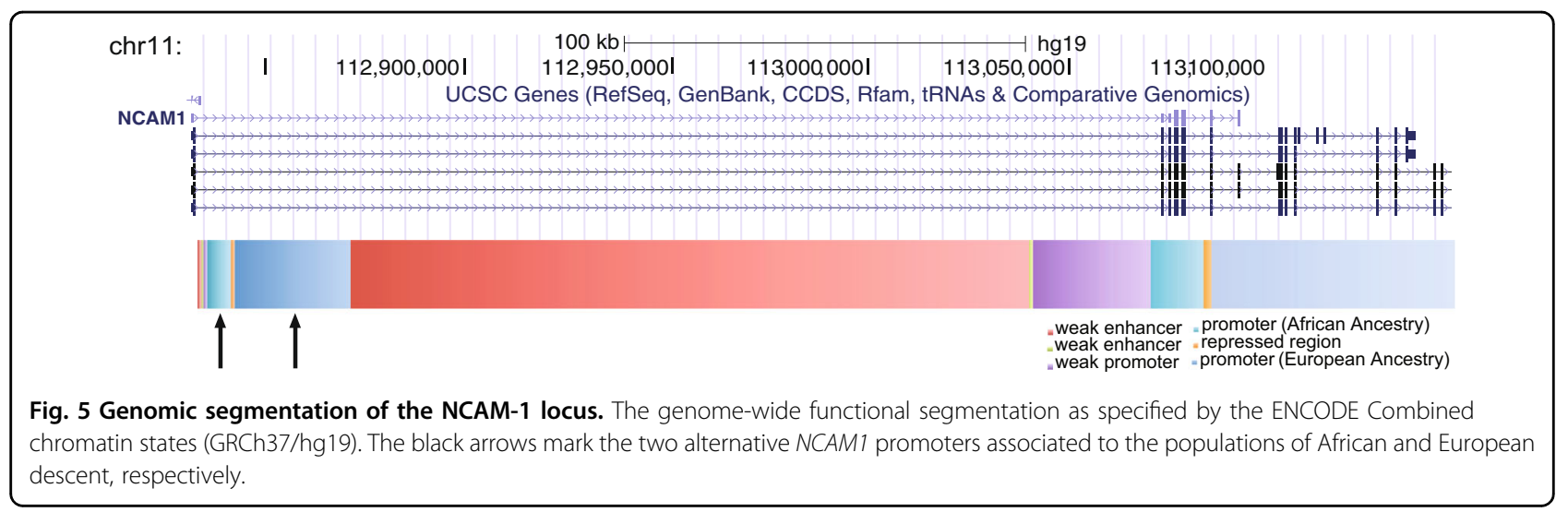

Table 1 Associations of putative NCAM1 promoter methylation with delayed recognition and delayed recall memory in Swiss samples of healthy young individuals.

\begin{tabular}{llll}
\hline Sample & Valence & $\boldsymbol{t}$-stat & $\boldsymbol{p}$ Value \\
\hline Swiss sample 1 & All pictures & -3.6 & 0.0004 \\
Delayed recognition & Negative pictures & -1.9 & 0.041 \\
Memory & Positive pictures & -0.9 & 0.359 \\
$(\mathrm{~N}=568)$ & Neutral pictures & -2.9 & 0.004 \\
Swiss sample 2 & All pictures & -3.0 & 0.003 \\
24h delayed recall & Negative pictures & -2.7 & 0.006 \\
Memory & Positive pictures & -1.8 & 0.077 \\
$(\mathrm{~N}=319)$ & Neutral pictures & -0.6 & 0.572 \\
\hline
\end{tabular}

$P=0.003, P_{\text {corrected }}<0.05$; and African sample 2: $t_{(460)}=$ $-3.3, P=0.001)$, after taking trauma load into account. Specifically, after accounting for the experienced traumatic event types, the DNA methylation of this putative NCAM1 promoter was negatively associated with lifetime intrusive memories of aversive events in both conflict zone survivor populations. On the other hand, the association with lifetime avoidance symptoms was significant only in African sample 1 and with lifetime hyperarousal symptoms only in African sample 2 (Table 2). In both African samples trauma load was positively associated with life-time PDS symptoms, as expected $\left(P_{s}<0.05\right)$, but had no significant effects on NCAM1 promoter DNA methylation $\left(P_{s}>0.05\right)$.

Finally, we investigated whether methylation levels at the putative NCAM1 gene promoter affected the association between trauma load and lifetime PTSD risk (see Materials and methods). We found that higher methylation levels were significantly associated with a lower lifetime PTSD risk in African Sample $1\left(t_{(347)}=-2.3\right.$, Wald $x^{2}=4.3, P=0.019$; Fig. 6), while in the African
Table 2 Associations of putative NCAM1 promoter with PTSD symptoms and PTSD risk in African Samples of conflict zone survivors.

\begin{tabular}{llll}
\hline Sample & Phenotype & $\boldsymbol{t}$-stat & $\boldsymbol{p}$ value \\
\hline African sample 1 & Avoidance & -2.4 & 0.019 \\
$(\mathrm{~N}=350)$ & Intrusions & -2.1 & 0.001 \\
& Hyperarousal & -0.5 & 0.601 \\
& Sum & -3.0 & 0.003 \\
& PTSD & -2.3 & 0.019 \\
African sample 2 & Avoidance & -1.7 & 0.089 \\
(N=463) & Intrusions & -2.7 & 0.029 \\
& Hyperarousal & -3.5 & 0.0005 \\
& Sum & -3.3 & 0.001 \\
& PTSD & -0.9 & 0.118 \\
\hline
\end{tabular}

sample 2 the association did not reach significance threshold $\left(t_{(460)}=-0.9, P=0.12\right.$; Table 2$)$.

Quantitative trait loci correlate with the variation of DNA methylation at the predicted NCAM1 promoter regions

Analysis of the methylation QTLs (meQTL) associated with the NCAM1 enhancer element methylation in the four cohorts revealed several in cis SNPs that significantly correlated with the variation of DNA methylation at the corresponding NCAM1 promoter regions. The Supplemental Tables S2 and S3 summarize the shared meQTLs across all the samples of European and African descent, respectively.

\section{Discussion}

The present study indicates that the neural cell adhesion molecule-1 is associated with associative long-term memory in nematodes and healthy humans, as well as with traumatic memories and PTSD risk in heavily traumatized conflict zone survivors. 


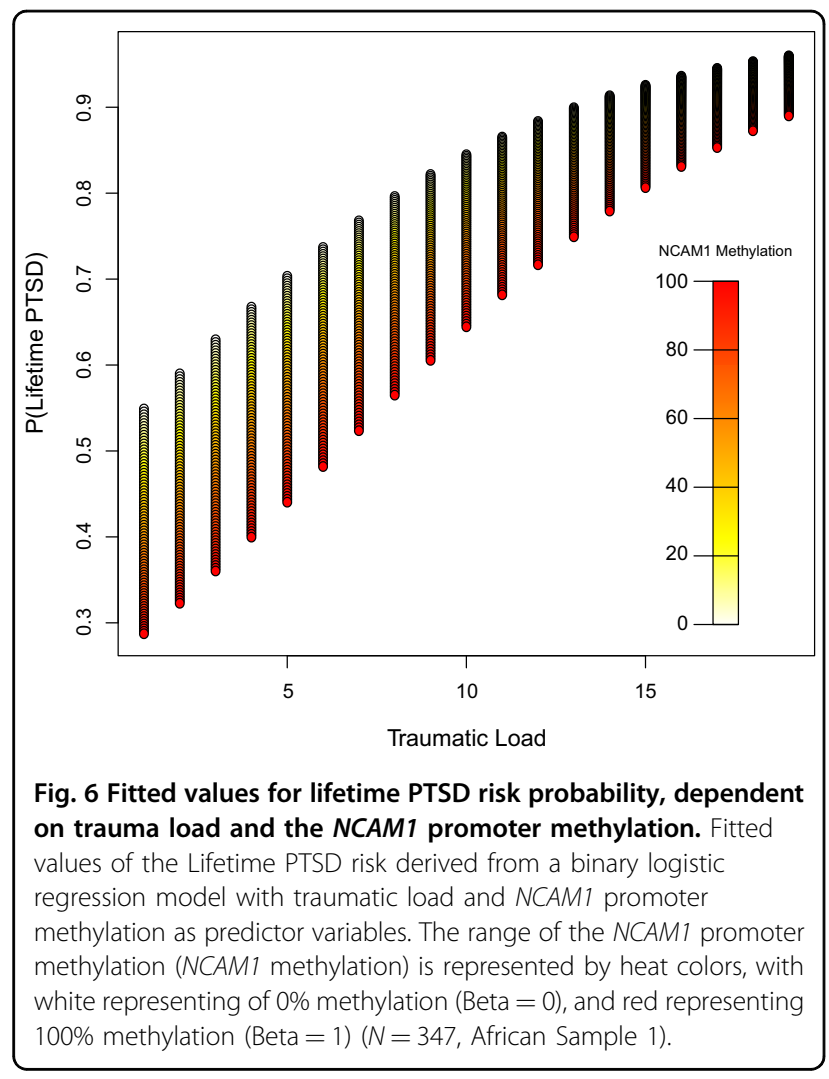

Phenotypic analysis of the neural cell adhesion molecule-1 (ncam-1) deficient worms demonstrated a selective role of ncam-1 for LTAM but not for learning (acquisition) or STAM. Previous studies in vertebrates have shown that NCAM1 is also essential in learning and short-term plasticity, particularly during the initiation phase of $\mathrm{LTP}^{4,12,13}$. However, the requirement of NCAM1 during learning has been mainly investigated in other model organisms using hippocampus-dependent tasks $^{52,53}$, which would explain why in C. elegans NCAM-1 is not involved in memory acquisition.

Besides the role of NCAM1 in the learning process, antibody-driven inhibition of NCAM1 during the proteinsynthesis-dependent phase of memory consolidation disrupts also the memory of the training experience ${ }^{54,55}$. Interestingly, a crucial period of $6-8 \mathrm{~h}$ post-passiveavoidance training is required for memory consolidation, which is in accord with the peak of transcriptional activation of ncam-1, $4 \mathrm{~h}$ after aversive olfactory conditioning training. Furthermore, ncam-1 remained upregulated even $24 \mathrm{~h}$ after post-conditioning. These results suggest that transcriptional activation of NCAM-1 in the first $4-6 \mathrm{~h}$ could be important for long-term memory formation. Upregulation of NCAM-1 at the protein level following aversive olfactory conditioning would provide additional evidence, however, given the high endogenous expression of NCAM-1 in the pharyngeal tissue of the worms (Fig. 3b, c), we were unable to detect any changes coming from neural tissues (Supplemental Fig. S3). This was also paralleled by imaging results, where differences from the VNC, where most synapses are located, could not be detected due to high baseline signal (Supplemental Fig. S3).

Complex mechanisms are known to underlie memory both on the molecular as well as the anatomical level. In addition, precise temporal control of such processes is crucial for memory formation. Although our results (Fig. 1) suggest that transcriptional activation of NCAM-1 likely regulates its function during memory formation, we were not able to address the exact time window where such processes are essential.

The cellular function of NCAMs is to fine-tune the adhesive properties of cells and regulate the establishment of the cellular connectivity pattern in the brain $^{7,56,57}$. During neurite outgrowth cells increase their migratory capacity by downregulating expression of cell adhesion molecules whilst once they innervate their target brain areas, their adhesiveness is upregulated, stabilizing the newly formed connections and locking cells into their final position ${ }^{58}$. Since NCAM1 expression is critical during nervous system development, memory deficits due to lack of NCAM1 could be related to the elimination of the protein during a critical developmental period and unrelated to its absence in adulthood. Indeed, by tagging the endogenous protein in C. elegans, NCAM-1 was expressed throughout the lifecycle of the worm, pointing to a continuous requirement for the gene, both in neuronal and nonneuronal tissues. These findings are supported by a previous report investigating the expression pattern of NCAM-1 with the help of a transcriptional reporter sys$t^{5} \mathrm{~m}^{50}$. However, given the strong functional redundancy between members of the IgCAM family ${ }^{50}$, loss of NCAM1 function during development might be compensated, leading to proper contact and communication among neurons during synaptogenesis. In line with that, our RNAi experiment revealed that the memory phenotype was not due to a developmental defect, as L3 worms whose nervous system is established with a ncam-1 knockdown phenocopied ncam-1 (lf). In addition, ionotropic glutamate-receptor (GLR-1) localization studies revealed no detectable deficits in synapse morphology or distribution.

Furthermore, given the sequence homology shared between $C$. elegans and human NCAM-1, the conserved functional role observed across species, as well as the fact that the introduction of the human homolog rescued the memory defect of ncam-1(lf) in worms, prompted us to investigate the role of NCAM-1 in human memory.

In the present study we show that epigenetic signatures of the NCAM1 gene promoter were associated with episodic memory performance in two independent samples of European descent. Moreover, in two populations of 
conflict zone survivors of African descent, DNA methylation at an alternative, putative promoter of the NCAM1 gene was negatively correlated with lifetime intrusive memories of aversive events, after taking trauma load into account. Interestingly, interindividual differences in DNA methylation levels were not related to traumatic load in traumatized individuals. Furthermore, we did not observe significant differences in median, distribution, or variability of NCAM1 promoter methylation between conflict zone survivors and healthy individuals nonexposed to trauma. These findings suggest that NCAM1 methylation differences might pre-exist the conflict-related traumatic events. The pre-existing differences in DNA methylation may be a result of interplay between selected types of early adversity and specific timing of exposure ${ }^{59,60}$, may reflect other types of risks associated with the HPA axis inhibition $^{61,62}$, or alternatively might be a result of development/early life associated stochastic epigenetic variation $^{63}$. The profound role of NCAM1 for neuronal development during the perinatal period of life might offer a plasticity window that could significantly impact how we process and store experiences later in life. However, underpinning the casual pathways of early set epigenetic modifications, as well as their relative stability and reversibility requires further research.

Recently, we have shown that epigenetic regulation of glucocorticoid receptor gene is associated with memory processing in healthy individuals, but also plays an important role for the risk and pathogenesis of PTSD risk $^{24}$. Glucocorticoid signaling is a key mediator of the effects of stressful learning on long-term memory facilitation by modulation of the consolidation processes. In synergy with processes initiated by learning, stress and glucocorticoid signaling transiently activates the expression of NCAM in memory-associated brain regions, thus facilitating the formation of long-term memories ${ }^{64}$. As mentioned, NCAM1 is involved in acquisition and formation of emotional memories (reviewed in ref. ${ }^{6}$ ) and accordingly, is dysregulated in several neuropsychiatric disorders, such as schizophrenia, mood and anxiety disorders, or Alzheimer's disease ${ }^{65}$.

In the present study, DNA was isolated from whole blood in the Swiss samples and from saliva in the African samples. Although the blood cells are of mesodermal origin, and the source of DNA in saliva is a mixture of ectodermal epithelial cells and mesodermal white blood cells $^{66}$, we did not observe significant differences in the NCAM1 methylation levels across the four samples investigated in this study. In addition, in a subset of European individuals with available DNA both from saliva and blood, NCAM1 methylation did not significantly differ across the tissues ${ }^{44}$. Previous studies suggested that DNA methylation in CG-rich genomic regions (e.g., NCAM1 promoter) generally show more stable epigenetic signatures across brain and non-brain tissues ${ }^{67-71}$. Finally, in the current study, several in cis meQTLs were associated with epigenetic signatures of the predicted NCAM1 promoters, thus suggesting that these epigenetic marks share a degree of similarity across the tissues. These findings suggest that it might be possible, to a certain extent, to use non-brain tissue for the investigation of brain-related traits, such as psychiatric disorders.

Taken together, our results support a conserved role of NCAM1 in memory from nematodes to humans. This knowledge might prove helpful in elucidating novel therapies in the treatment of memory-related disorders.

\section{Acknowledgements}

This work was supported by the Swiss National Science Foundation (Grant 159740 to D.J.-F.D.Q.) and by the German Research Foundation (grants to I.-T.K. and T.E.). We are grateful to Anne Spang for generously sharing reagents and instruments. We would also like to thank the Caenorhabditis Genetic Center (supported by NIH-NCRR) for providing nematode strains as well as the Imaging Core Facility (IMCF).

\section{Author details}

'University of Basel, Department of Psychology, Division of Molecular Neuroscience, Birmannsgasse 8, CH-4055 Basel, Switzerland. ${ }^{2}$ University of Basel, Department Biozentrum, Life Sciences Training Facility,

Klingelbergstrasse 50-70, CH-4056 Basel, Switzerland. ${ }^{3}$ University of Basel, Psychiatric University Clinics, Wilhelm Klein-Strasse 27, CH-4012 Basel, Switzerland. ${ }^{4}$ University of Basel, Transfaculty Research Platform, Birmannsgasse 8, CH-4055 Basel, Switzerland. ${ }^{5}$ Ulm University, Clinical \& Biological Psychology, Institute for Psychology \& Education, Albert-Einstein-Allee 47, D-89069 Ulm, Germany. ${ }^{6}$ University Bielefeld, Department for Psychology and Sports Science, P.O. Box 100131D-33501 Bielefeld, Germany. ${ }^{7}$ University of Konstanz, Clinical Psychology \& Behavioural Neuroscience, D-78457 Konstanz, Germany.

${ }^{8}$ University of Basel, Department of Psychology, Division of Cognitive Neuroscience, Birmannsgasse 8, CH-4055 Basel, Switzerland

\section{Conflict of interest}

The authors declare that they have no conflict of interest.

\section{Publisher's note}

Springer Nature remains neutral with regard to jurisdictional claims in published maps and institutional affiliations.

Supplementary Information accompanies this paper at (https://doi.org/ 10.1038/s41398-020-00899-y).

Received: 9 October 2019 Revised: 11 June 2020 Accepted: 16 June 2020 Published online: 06 July 2020

\footnotetext{
References

1. Alberini, C. M. \& Kandel, E. R. The regulation of transcription in memory consolidation. Cold Spring Harb. Perspect. Biol. 7, a021741 (2014).

2. Benito, E. \& Barco, A. The neuronal activity-driven transcriptome. Mol. Neurobiol. 51, 1071-1088 (2015).

3. Martin, S. J., Grimwood, P. D. \& Morris, R. G. Synaptic plasticity and memory: an evaluation of the hypothesis. Annu Rev. Neurosci. 23, 649-711 (2000).

4. Kiss, J. Z., Troncoso, E., Djebbara, Z., Vutskits, L. \& Muller, D. The role of neural cell adhesion molecules in plasticity and repair. Brain Res. Brain Res. Rev. 36, 175-184 (2001).

5. Venero C., Herrero A. I., Touyarot K, Cambon K., Sandi C. Hippocampal upregulation of NCAM expression and polysialylation plays a key role on spatial memory. Eur. J. Neurosci. 23, 1585-1595 (2006).

6. Conboy, L., Bisaz, R., Markram, K. \& Sandi, C. Role of NCAM in emotion and learning. Adv. Exp. Med. Biol. 663, 271-296 (2010).
} 
7. Muller, D. et al. Role of NCAM in spine dynamics and synaptogenesis. Adv. Exp. Med. Biol. 663, 245-256 (2010).

8. Togashi, H., Sakisaka, T. \& Takai, Y. Cell adhesion molecules in the central nervous system. Cell Adhes. Migr. 3, 29-35 (2009).

9. Cunningham, B. et al. Neural cell adhesion molecule: structure, immunoglobulin-like domains, cell surface modulation, and alternative RNA splicing. Science 236, 799-806 (1987).

10. Walmod, P. S., Kolkova, K., Berezin, V. \& Bock, E. Zippers make signals: NCAMmediated molecular interactions and signal transduction. Neurochem. Res. 29 , 2015-2035 (2004).

11. Goridis, C. \& Brunet, J. F. NCAM: structural diversity, function and regulation of expression. Semin. Cell Biol. 3, 189-197 (1992).

12. Fields D. R., Kouichi I. Neural cell adhesion molecules in activity-dependent development and synaptic plasticity. Trends Neurosci. 19, 473-480 (1996).

13. Schachner M. Neural recognition molecules and synaptic plasticity. Curr. Opin. Cell Biol. 9, 627-634.

14. Luthl, A., Laurent, J. P., Figurov, A.r Muller, D. \& Schachner, M. Hippocampal long-term potentiation and neural cell adhesion molecules L1 and NCAM. Nature 372, 777-779 (1994).

15. Cremer, $\mathrm{H}$. et al. Inactivation of the N-CAM gene in mice results in size reduction of the olfactory bulb and deficits in spatial learning. Nature $\mathbf{3 6 7}$, 455-459 (1994).

16. Stork, O. et al. Recovery of emotional behaviour in neural cell adhesion molecule (NCAM) null mutant mice through transgenic expression of NCAM180. Eur. J. Neurosci. 12, 3291-3306 (2000).

17. Senkov, O. et al. Polysialylated neural cell adhesion molecule is involved in induction of long-term potentiation and memory acquisition and consolidation in a fear-conditioning paradigm. J. Neurosci. 26, 10888-109898 (2006).

18. Stork, O., Welzl, H., Cremer, H. \& Schachner, M. Increased intermale aggression and neuroendocrine response in mice deficient for the neural cell adhesion molecule (NCAM). Eur. J. Neurosci. 9, 1117-1125 (1997).

19. Sandi, C. Acute stress-induced impairment of spatial memory is associated with decreased expression of neural cell adhesion molecule in the hippocampus and prefrontal cortex. Biol. Psychiatry 57, 856-864 (2005).

20. Freytag, V. et al. Genome-wide temporal expression profiling in caenorhabditis elegans identifies a core gene set related to long-term memory. J. Neurosci. 37, 6661-6672 (2017).

21. Barton, C. H., Mann, D. A. \& Walsh, F. S. Characterization of the human N-CAM promoter. Biochem. J. 268, 161-168 (1990).

22. Holliday R. Is there an epigenetic component in long-term memory? J. Theor. Biol. 200, 339-341.

23. Atucha, E. et al. Noradrenergic activation of the basolateral amygdala maintains hippocampus-dependent accuracy of remote memory. Proc. Natl Acad. Sci. USA 114, 9176-9181 (2017)

24. Vukojevic, V. et al. Epigenetic modification of the glucocorticoid receptor gene is linked to traumatic memory and post-traumatic stress disorder risk in genocide survivors. J. Neurosci. 34, 10274-10284 (2014).

25. Guo, J. U. et al. Neuronal activity modifies the DNA methylation landscape in the adult brain. Nat. Neurosci. 14, 1345-1351 (2011).

26. Miller, C. A. \& Sweatt, J. D. Covalent modification of DNA regulates memory formation. Neuron 53, 857-869 (2007).

27. Lubin, F. D. Epigenetic gene regulation in the adult mammalian brain: multiple roles in memory formation. Neurobiol. Learn. Mem. 96, 68-78 (2011).

28. Brenner, S. The genetics of Caenorhabditis elegans. Genetics 77, 71-94 (1974).

29. Bargmann, C. I., Hartwieg, E. \& Horvitz, H. R. Odorant-selective genes and neurons mediate olfaction in C. elegans. Cell 74, 515-527 (1993).

30. Nuttley, W. M., Atkinson-Leadbeater, K. P. \& Van Der Kooy, D. Serotonin mediates food-odor associative learning in the nematode Caenorhabditis elegans. Proc. Natl Acad. Sci. USA 99, 12449-12454 (2002).

31. Vukojevic, $V$. et al. A role for $a$-adducin (ADD-1) in nematode and human memory. EMBO J. 31, 1453-1466 (2012).

32. Pfaffl, M. W. A new mathematical model for relative quantification in real-time RT-PCR. Nucleic Acids Res. 29, e45 (2001).

33. de Quervain, D. J. -F. et al. A deletion variant of the alpha2b-adrenoceptor is related to emotional memory in Europeans and Africans. Nat. Neurosci. 10, 1137-1139 (2007).

34. Heck, A. et al. exome sequencing of healthy phenotypic extremes links TROVE2 to emotional memory and PTSD. Nat. Hum. Behav. 1, 0081 (2017).

35. Association A. P. Diagnostical and Statistical Manual of Mental Disorders, Fourth ed. (DSM-IV-TR) (American Psychiatric Association, Washington, DC, 2000).
36. Wilker, S. et al. How to quantify exposure to traumatic stress? Reliability and predictive validity of measures for cumulative trauma exposure in a postconflict population. Eur. J. Psychotraumatol. 6, 28306 (2015).

37. Kolassa, I. T. et al. Association study of trauma load and SLC6A4 promoter polymorphism in posttraumatic stress disorder: evidence from survivors of the Rwandan genocide. J. Clin. Psychiatry 71, 543-547 (2010).

38. Assenov, Y. et al. Comprehensive analysis of DNA methylation data with RnBeads. Nat. Methods 11, 1138-1140 (2014).

39. Davis S, Du P, Bilke S, Triche, JrT, Bootwalla M. methylumi: Handle Illumina methylation data. R package version 2.34.0. (2020).

40. Maksimovic, J., Gordon, L. \& Oshlack, A. SWAN: subset-quantile within array normalization for illumina infinium HumanMethylation450 BeadChips. Genome Biol. 13, R44 (2012).

41. Dunham, I. et al. An integrated encyclopedia of DNA elements in the human genome. Nature 489, 57-74 (2012).

42. Hoffman, M. M. et al. Unsupervised pattern discovery in human chromatin structure through genomic segmentation. Nat. Methods 9, 473-476 (2012).

43. Lawrence, M., Gentleman, R. \& Carey, V. rtracklayer: an R package for interfacing with genome browsers. Bioinformatics 25, 1841-1842 (2009).

44. Milnik, A. et al. Common epigenetic variation in a European population of mentally healthy young adults. J. Psychiatr. Res. 83, 260-268 (2016).

45. Barfield, R. T., Kilaru, V., Smith, A. K. \& Conneely, K. N. CpGassoc: an R function for analysis of DNA methylation microarray data. Bioinformatics 28, 1280-1281 (2012).

46. Pinheiro J, Bates D, DebRoy S, Sarkar D, R Core Team. nIme: Linear and Nonlinear Mixed Effects Models. R package version 3.1-148 (2020).

47. Lakhina, V. et al. Genome-wide functional analysis of CREB/long-term memory-dependent transcription reveals distinct basal and memory gene expression programs. Neuron 85, 330-345 (2015).

48. Bernhard, N. \& van der Kooy, D. A behavioral and genetic dissection of two forms of olfactory plasticity in Caenorhabditis elegans: adaptation and habituation. Learn. Mem. 7, 199-212 (2000).

49. Stetak, A., Hörndli, F., Maricq, A. V., Van Den Heuvel, S. \& Hajnal, A. Neuronspecific regulation of associative learning and memory by MAGI-1 in C. elegans. PLOS ONE 4, e6019 (2009).

50. Schwarz, V., Pan, J., Voltmer-Irsch, S. \& Hutter, H. IgCAMs redundantly control axon navigation in Caenorhabditis elegans. Neural Dev. 4, 13 (2009).

51. Morrison, G. E. \& van der Kooy, D. A mutation in the AMPA-type glutamate receptor, glr-1, blocks olfactory associative and nonassociative learning in Caenorhabditis elegans. Behav. Neurosci. 115, 640-649 (2001).

52. Blozovski, D. \& Harris, P. Passive avoidance deficits following lesions of the posteroventral hippocampo-subiculo-entorhinal area in the developing rat. J. Physiol. 81, 374-378 (1986).

53. Moser, D. et al. The glucocorticoid receptor gene exon 1-F promoter is not methylated at the NGFI-A binding site in human hippocampusn. World J. Biol. Psychiatry 8, 262-268 (2007).

54. Doyle, E., Nolan, P. M., Bell, R. \& Regan, C. M. Intraventricular infusions of antineural cell adhesion molecules in a discrete posttraining period impair consolidation of a passive avoidance response in the rat. J. Neurochem. 59, 1570-1573 (1992).

55. Rose, S. P. R. Cell-adhesion molecules, glucocorticoids and long-term-memory formation. Trends Neurosci. 18, 502-506 (1995).

56. Chuong, C. M. \& Edelman, G. M. Alterations in neural cell adhesion molecules during development of different regions of the nervous system. J. Neurosci. 4, 2354-2368 (1984).

57. Rougon, G. \& Hobert, O. New insights into the diversity and function of neuronal immunoglobulin superfamily molecules. Annu. Rev. Neurosci. 26, 207-238 (2003).

58. Edelman, G. M. \& Crossin, K. L. Cell adhesion molecules: implications for a molecular histology. Annu. Rev. Biochem. 60, 155-190 (1991).

59. Oberlander, T. F. et al. Prenatal exposure to maternal depression, neonatal methylation of human glucocorticoid receptor gene (NR3C1) and infant cortisol stress responses. Epigenetics 3, 97-106 (2008).

60. Mcgowan, P. O. et al. Epigenetic regulation of the glucocorticoid receptor in human brain associates with childhood abuse. Nat. Neurosci. 12, 342-348 (2009).

61. Yehuda, R. Status of glucocorticoid alterations in post-traumatic stress disorder. Ann. N. Y. Acad. Sci. 1179, 56-69 (2009).

62. Yehuda, R. et al. Gene expression patterns associated with posttraumatic stress disorder following exposure to the World Trade Center attacks. Biol. Psychiatry 66, 708-711 (2009) 
63. Feinberg, A. P. \& Irizarry, R. A. Stochastic epigenetic variation as a driving force of development, evolutionary adaptation, and disease. Proc. Natl Acad. Sci. USA 107, 1757-1764 (2010).

64. Bisaz, R., Conboy, L. \& Sandi, C. Learning under stress: a role for the neural cell adhesion molecule NCAM. Neurobiol. Learn. Mem. 91, 333-342 (2009).

65. Brennaman, L. H. \& Maness, P. F. NCAM in Neuropsychiatric and Neurodegenerative Disorders. Structure and Function of the Neural Cell ..., Advances in Experimental Medicine and Biology. (pp. 299-317. Springer, New York, New York, NY, 2009).

66. Zhou, Y. et al. DNA profiling in blood, buccal swabs and hair follicles of patients after allogeneic peripheral blood stem cells transplantation. Leg. Med. 13, 47-51 (2011).
67. Ladd-Acosta, C. et al. DNA methylation signatures within the human brain Am. J. Hum. Genet. 81, 1304-1315 (2007).

68. Mill, J. et al. Epigenomic profiling reveals DNA-methylation changes associated with major psychosis. Am. J. Hum. Genet. 82, 696-711 (2008).

69. Lister, R. et al. Human DNA methylomes at base resolution show widespread epigenomic differences. Nature 462, 315-322 (2009).

70. Dempster, E. L. et al. Disease-associated epigenetic changes in monozygotic twins discordant for schizophrenia and bipolar disorder. Hum. Mol. Genet. 20 4786-4796 (2011).

71. Davies, M. N. et al. Functional annotation of the human brain methylome identifies tissue-specific epigenetic variation across brain and blood. Genome Biol. 13, R43 (2012). 